\title{
On global well-posedness for the Einstein-Maxwell-Euler system in Bondi coordinates
}

\author{
Mamadou Sango $(*)$ - Calvin Tadmon $(* *)$
}

ABSTRACT - We analyze the Einstein-Maxwell equations for an irrotational stiff fluid. Under the spherical symmetry assumption on the space-time, in Bondi coordinates, the considered model is reduced to a nonlinear evolution system of partial integrodifferential equations. Assuming regularity at the center of symmetry and that the matter content of the initial light cone is the so-called null dust, the characteristic initial value problem associated to the obtained system is solved globally by a contraction mapping argument. In future work we will address the issue of global well-posedness for the considered model in other physically interesting cases where the matter content of the initial light cone is not the null dust.

Mathematics Subject Classification (2010). 35A01, 35A02, 35A09, 35B06, 35Q31, 45K05, 76W05, 83C22.

KeYwords. Characteristic Cauchy problem, Einstein-Maxwell-Euler equations, spherical symmetry, irrotational perfect fluid, Bondi coordinates

\section{Introduction}

Since their formulation, the relativistic theories of gravitation, electromagnetism and hydrodynamics have experienced an intensive and rapidly developing research activity over the years. The system that unifies these three theories consists of the Einstein-Maxwell-Euler (EME) equations. These equations are of central importance in several areas of high-energy

(*) Indirizzo dell'A.: Department of Mathematics and Applied Mathematics University of Pretoria, 0002 Pretoria, South Africa.

E-mail: mamadou.sango@up.ac.za

(**) Indirizzo dell'A.: Department of Mathematics and Computer Science University of Dschang, P.O. Box 67 Dschang, Cameroon.

E-mails: calvin.tadmon@up.ac.za_tadmone@yahoo.fr 
astrophysics, plasma physics and nuclear physics. We refer the reader to three excellent books by Lichnerowicz [1], Choquet-Bruhat [2] and Anile [3] for a thorough review on these equations and their interests, both on mathematical and physical view points. Here we briefly mention some areas and problems which constitute physical motivations for studying EME equations: collapse of massive rotating stars towards neutron stars or black holes, merging of binary systems, models of supernova explosions, structure and stability of neutron stars, galactic microquasars, active galactic nuclei, gamma-ray bursts, free electron laser, high-energy collisions among heavy nuclei. Apart from the fundamental in-depth mathematical analysis [1, 2, 3] of the EME equations, a couple of recent contributions can be found in $[4,5,6,7]$ and some references therein. It should be noted that few works have been devoted to the problem of global well-posedness for the EME equations. This is understandable since these equations are highly nonlinear and investigating their global well-posedness is a very difficult task. To make some progress in this direction, it is reasonable to work under some simplifying and physically interesting assumptions. Noutchegueme and Nangne [8] proved global existence of a unique solution to the EME system for a perfect fluid of pure radiation type in a Robertson-Walker space-time, by reducing the considered model to a nonlinear evolution system of ordinary differential equations (ODE). In the present investigation we address the issue of global existence and uniqueness for the EME equations, for an irrotational stiff fluid evolving in a spherically symmetric curved space-time. The investigation is conducted in the so-called Bondi coordinates so that the problem at hand is a characteristic initial value problem with initial data assigned on a light cone with tip at the center of symmetry. The importance of characteristic initial value problems (with initial data prescribed on one or more null hypersurfaces of the space-time) was amply demonstrated by Christodoulou [9] in his famous book on black holes formation. In Bondi coordinates, we reduce the problem to a nonlinear evolution system of partial integrodifferential equations (PIDE). The mathematical analysis of the obtained system faces two major difficulties: (1) the appearance of non-converging integrals of the form $\int_{r}^{\infty} \frac{d s}{s(1+u+s)^{m}}$ in some essential estimates, and (2) the treatment of initial data whose smallness does not necessarily ensure the existence and uniqueness of a solution for the considered problem. To overcome these difficulties we have defined an appropriate functional framework and assume that the matter content of the initial light cone is the so-called null dust. This 
latter assumption amounts to assigning zero initial data for the initial value problem associated to the obtained nonlinear evolution system (see (31)), which we solve globally by a fixed point method. The present work is the generalization, to the considered EME model, of previous ones achieved by Christodoulou [10] for the Einstein-scalar field system, Chae [11] for the Einstein-Maxwell-Higgs system, Tadmon and Tchapnda [12, 13] for the Einstein-Yang-Mills-Higgs system. Some work on characteristic initial value problems without symmetry assumption can be found in $[14,15,16]$.

The rest of the paper is outlined as follows. Section 2 is devoted to the derivation of the considered EME equations in Bondi coordinates. We show that the equations reduce to a nonlinear evolution system of PIDE. The main result of the work is stated and proved in section 3 .

\section{Electrovacuum Einstein-Maxwell-Euler equations in Bondi coor- dinates}

\section{1 - General form of the Einstein-Maxwell-Euler equations}

The evolution of a charged perfect fluid is governed by the EinsteinMaxwell-Euler equations which, in local chart $\left(x^{\alpha}\right)_{\alpha=0, \ldots, 3}$ on a four dimensional space-time manifold, read

$$
\begin{gathered}
R_{\alpha \beta}-\frac{1}{2} g_{\alpha \beta} R=T_{\alpha \beta}, \\
\nabla_{\alpha}(* F)^{\alpha \beta}=0, \quad \nabla_{\alpha} F^{\alpha \beta}=J^{\beta}, \\
\nabla_{\alpha} T^{\alpha \beta}=0 .
\end{gathered}
$$

(1) are the Einstein equations where $g_{\alpha \beta}$ are the components of the unknown space-time metric $\mathbf{g}$ which, throughout the work, is assumed to have signature $(-,+,+,+) \cdot R_{\alpha \beta}$ and $R$ represent, respectively, the Ricci tensor and the scalar curvature relative to the space-time metric. $T_{\alpha \beta}$ are the components of the energy-momentum tensor, given by

$$
T_{\alpha \beta}=(p+\rho) U_{\alpha} U_{\beta}+p g_{\alpha \beta}+g^{\lambda \mu} F_{\alpha \lambda} F_{\beta \mu}-\frac{1}{4} g_{\alpha \beta} F_{\gamma \delta} F^{\gamma \delta},
$$

where $p$ is the pressure of the fluid, $\rho$ its mass-energy density, and $\mathbf{U}$ its four velocity normalized to unity so that $U_{\alpha} U^{\alpha}=-1$. (2) consists, respectively, of the first and second groups of Maxwell equations, where $\nabla$ denotes 
covariant derivative w.r.t. the space-time metric. $F^{\alpha \beta}$ are the contravariant components of the unknown antisymmetric electromagnetic field (also called Faraday tensor) $\mathbf{F} ; * \mathbf{F}$ is the Hodge dual of $\mathbf{F} . J^{\beta}$ are the components of the charge-current four-vector $\mathbf{J}$, which classically splits into convection part and conduction part as $J^{\beta}=\delta U^{\beta}+\sigma e^{\beta}$, where $\delta$ is the density of electric charge in the fluid, $\sigma$ the electrical conductivity of the fluid, and $\mathbf{e}$ is the four-vector electric field defined by $e^{\alpha}=U_{\beta} F^{\beta \alpha}$. (3) are the relativistic Euler equations.

\section{2 - Electrovacuum Einstein-Maxwell-Euler system}

It will be assumed from now on that the density of electric charge and the electrical conductivity of the fluid vanish identically:

$$
\delta \equiv 0, \quad \sigma \equiv 0 .
$$

The charge-current four-vector $\mathbf{J}$ then vanishes identically, i.e,

$$
\mathbf{J} \equiv 0 .
$$

The EME system for a fluid with zero density of electric charge and zero electrical conductivity is often referred to as electrovac EME system. It consists of twenty three equations, including equation $U_{\alpha} U^{\alpha}=-1$, with twenty two unknowns $\left(10 g_{\alpha \beta}, 4 U_{\alpha}, 6 F_{\alpha \beta}, p, \rho\right)$. An equation of state is needed to close it. Moreover it is a highly nonlinear system and, in order to obtain global solution for it, it makes sense to impose some more simplifying assumptions. As in $[10,11,12,13]$ we will work in a Bondi coordinates system $\left(x^{\alpha}\right)=(u, r, \theta, \varphi)$, where the spherically symmetric space-time metric takes the form

$$
g_{\alpha \beta \beta} d x^{\alpha} d x^{\beta}=-e^{2 v} d u^{2}-2 e^{v+\lambda} d u d r+r^{2}\left(d \theta^{2}+\sin ^{2} \theta d \varphi^{2}\right) .
$$

Here $v$ and $\lambda$ are real functions of the retarded time coordinate $u$ and the radial coordinate $r$ only. We assume the flow to be stiff and irrotational. Then the equation of state reads

$$
p=\rho,
$$

and the four velocity of the fluid can be written as

$$
U_{\alpha}=\left(-\psi_{, \mu} \psi^{, \mu}\right)^{-\frac{1}{2}} \psi_{, \alpha},
$$

where $\psi$ is a time function, i.e $\psi_{, \mu} \psi^{, \mu}<0$; the subscript "," denotes usual 
partial derivative. Supposing that $\rho>0$, after some algebra, it follows that

$$
\begin{gathered}
\rho=-\psi{ }_{, \mu} \psi^{, \mu}, \\
\left(\psi^{, \alpha}\right)_{; \alpha}=0,
\end{gathered}
$$

where the subscript "," denotes covariant derivative. It is worth mentioning that (10) is the relativistic counterpart of the famous Bernoulli's law for irrotational flows in classical Fluids Mechanics, and it determines $\rho$ (and hence $p$ ) once the potential $\psi$ and the space-time metric $\mathbf{g}$ are known.

Note that equations (10) and (11) above have been obtained under the condition $\psi_{, \mu} \psi^{, \mu}<0$; in that case, using (8), (9) and (10), the energy-momentum tensor in (4) becomes

$$
T_{\alpha \beta}=2 \psi_{, \alpha} \psi_{, \beta}-\psi_{, \mu} \psi^{, \mu} g_{\alpha \beta}+g^{\lambda \mu} F_{\alpha \lambda} F_{\beta \mu}-\frac{1}{4} g_{\alpha \beta} F_{\gamma \delta} F^{\gamma \delta},
$$

which is regular and well defined irrespective of the sign of $\psi_{, \mu} \psi^{, \mu}$. The cases $\psi_{, \mu} \psi^{, \mu}>0$ and $\psi_{, \mu} \psi^{, \mu}=0$, can be interpreted by arguments similar to those used in $[17,18]$. When $\psi_{, \mu} \psi^{, \mu}=0$, the energy-momentum tensor defined by (12) reduces to

$$
T_{\alpha \beta}=2 \psi_{, \alpha} \psi_{, \beta}+g^{\lambda \mu} F_{\alpha \lambda} F_{\beta \mu}-\frac{1}{4} g_{\alpha \beta} F_{\gamma \delta} F^{\gamma \delta} .
$$

If $\frac{\partial \psi}{\partial r}$ and $\mathbf{F}$ vanish identically, (13) coincides with the energy-momentum tensor of the so-called null dust matter. This will serve as the matter content of the light cone where initial data will be specified. From now on $\psi$ is regarded as the main fluid variable, whose evolution is governed by equation (11), and (12) as the expression of the energy-momentum tensor.

We will use the following ansatz for the electromagnetic field F, compatible with the ansatz (7) for the space-time metric (this facilitates calculation of the components of the energy-momentum tensor (13)):

$$
\left(F_{\alpha \beta}\right)=\left(\begin{array}{cccc}
0 & 0 & a & b \sin \theta \\
0 & 0 & k & l \sin \theta \\
-a & -k & 0 & 0 \\
-b \sin \theta & -l \sin \theta & 0 & 0
\end{array}\right),
$$

where $a, b, k$, and $l$ are functions of $u$ and $r$ only. Using (14), direct calculation shows that the first group of Maxwell equations in (2) are equivalent to

$$
\dot{k}=a^{\prime}, \quad \dot{l}=b^{\prime} .
$$


Here and in the remainder of the work, - denotes differentiation with respect to $u$ and ' differentiation with respect to $r$. Similarly, using (5), (6), (7) and (14), one deduces from straightforward though lengthy computation that the second group of Maxwell equations in (2) are satisfied if and only if

$$
\begin{gathered}
a=k=0, \\
\left(\dot{l}+b^{\prime}\right) e^{-v-\lambda}-l^{\prime} e^{-2 \lambda}=l\left(v^{\prime}-\lambda^{\prime}\right) e^{-2 \lambda} .
\end{gathered}
$$

Observe that the first equation in (15) is satisfied if it is the case for (16); if the second equation in (15) is satisfied then equation (17) becomes

$$
\dot{l}-\frac{1}{2} e^{v-\lambda} l^{\prime}=\frac{1}{2}\left(e^{v-\lambda}\right)^{\prime} l .
$$

We now handle the Einstein equations (1). As in $[10,11,12,13]$ we introduce an adapted null tetrad $\left(e_{\alpha}\right)_{\alpha=0, \ldots, 3}$ defined by $e_{0}=e^{-v} \frac{\partial}{\partial u}-\frac{1}{2} e^{-\lambda} \frac{\partial}{\partial r}$, $e_{1}=e^{-\lambda} \frac{\partial}{\partial r},\left(e_{2}, e_{3}\right)$ is an orthonormal frame on the unit $2-$ sphere. After lengthy calculation the relevant Einstein fields equations are found to be equivalent to the following:

$$
\begin{gathered}
\frac{1}{2}\left(\lambda^{\prime}+v^{\prime}\right)-2 e^{\lambda-v} \dot{\lambda}=2 r e^{2 \lambda}\left(e^{-v} \dot{\psi}-\frac{1}{2} e^{-\lambda} \psi^{\prime}\right)^{2}+r^{-1} e^{2 \lambda}\left(e^{-v} b-\frac{1}{2} e^{-\lambda} l\right)^{2} \\
\left(e^{v-\lambda}\right)^{\prime}+r^{-1}\left(e^{v-\lambda}-e^{v+\lambda}\right)=0 \\
\lambda^{\prime}+v^{\prime}=r\left[\left(\psi^{\prime}\right)^{2}+\frac{1}{2} r^{-2} l^{2}\right] \\
v^{\prime \prime}+\left(v^{\prime}-\lambda^{\prime}\right)\left(v^{\prime}+r^{-1}\right)-e^{\lambda-v}\left[(\dot{\lambda})^{\prime}+(\dot{v})^{\prime}\right]=2 e^{\lambda-v} \dot{\psi} \psi^{\prime}-\left(\psi^{\prime}\right)^{2} \\
e^{-v-\lambda} b l-\frac{1}{2} e^{-2 \lambda} l^{2}=0
\end{gathered}
$$

Equation (11) reads

$$
2(\dot{\psi})^{\prime}+2 r^{-1} \dot{\psi}-e^{\nu-\lambda}\left[\psi^{\prime \prime}+\psi^{\prime}\left(2 r^{-1}+v^{\prime}-\lambda^{\prime}\right)\right]=0 .
$$

REMARK 1. (i) If $v, \lambda$, and $l$ are known, then setting

$$
b=\frac{1}{2} l e^{v-\lambda},
$$

it is easy to see that equation (23) is satisfied. Moreover the second equation $\dot{l}=b^{\prime}$ in (15) is equivalent to (18) for $b$ given by (25). 
(ii) If regularity is assumed at the center, an argument similar to the one used in $[10,13]$ leads to the conclusion that the Einstein equations $(20)$ and (21), together with equation (24) and (18), are equivalent to the full set of the considered electrovac EME equations.

2.3 - Reduction of the electrovac EME equations to a nonlinear evolution system

As in $[10,11,12,13]$ we define a new function $h=(r \psi)^{\prime}$ to gain

$$
\psi(u, r)=\bar{h}(u, r):=\frac{1}{r} \int_{0}^{r} h(u, s) d s, \quad \psi^{\prime}(u, r)=\frac{h(u, r)-\bar{h}(u, r)}{r} .
$$

In view of (26) the Einstein equation (21) reads $\lambda^{\prime}+v^{\prime}=\frac{1}{r}\left[(h-\bar{h})^{2}+\frac{1}{2} l^{2}\right]$, and the solution which satisfies the asymptotic condition $\lambda+v \longrightarrow 0$, as $r \longrightarrow \infty$, is

$$
\lambda+v=-\int_{r}^{+\infty} \frac{1}{s}\left[(h-\bar{h})^{2}+\frac{1}{2} l^{2}\right] d s .
$$

The Einstein equation (20) is integrated to give

$$
e^{\nu-\lambda}=\frac{1}{r} \int_{0}^{r} e^{\lambda+v} d s
$$

We use (26) to rewrite equation (24) as

$$
\dot{h}-\frac{1}{2} e^{v-\lambda} h^{\prime}=\frac{1}{2 r}\left(e^{\lambda+v}-e^{\nu-\lambda}\right)(h-\bar{h}) .
$$

Using the definition of $\bar{l}$ as in (26), we recast equation (18) as

$$
\dot{l}-\frac{1}{2} e^{v-\lambda} l^{\prime}=\frac{1}{2 r}\left(e^{\lambda+v}-e^{v-\lambda}\right)(l-\bar{l})+\frac{1}{2 r}\left(e^{\lambda+v}-e^{v-\lambda}\right) \bar{l} .
$$

In view of (26), (27) and (28), equations (29) and (30) form a nonlinear evolution system of PIDE. It should be noted that, in the spaces of functions used in previous work [10,11, 12, 13], the mathematical analysis of the model considered in the present investigation comes up against several challenges. One of these difficulties is to estimate $\int_{r}^{\infty} \frac{l^{2}}{s} d s$, which involves 
non-converging integrals of the form $\int_{r}^{\infty} \frac{d s}{s(1+u+s)^{m}}$. A way around this toughness is to define a new functional framework where all the relevant quantities can be estimated appropriately. Another delicate problem is the prescription of initial data for the considered EME system. In the present work we assume that the matter content on the light cone $u=0$ is the socalled null dust. This amounts to considering, as observed earlier in paragraph 2.2, the Cauchy problem for the aforementioned nonlinear evolution system of PIDE with zero initial data prescribed on the light cone $u=0$. Set $W=\left(\begin{array}{l}h \\ l\end{array}\right)$ and denote by $I$ the $2 \times 2$ identity matrix. Then, in view of (29) and (30), the problem under investigation reads

$$
\begin{aligned}
& D W(u, r)=\frac{1}{2 r}(g(u, r)-\widetilde{g}(u, r))[W(u, r)+(\sigma-I) \bar{W}(u, r)], \\
& W(0, r)=0
\end{aligned}
$$

where $D=\frac{\partial}{\partial u}-\frac{\widetilde{g}}{2} \frac{\partial}{\partial r}, \quad g=e^{\lambda+v}, \quad \widetilde{g}=e^{v-\lambda}, \quad \sigma=\left(\begin{array}{ll}0 & 0 \\ 0 & 1\end{array}\right)$.

\section{Existence and uniqueness of classical solutions}

We begin by introducing the spaces of functions used and some preliminary notations. For a vector function $W=\left(\begin{array}{l}h \\ l\end{array}\right)$ we will use the notation $|W|:=|h|+|l|$, and simply write $W \in C^{1}([0, \infty) \times(0, \infty))$ if $h$ and $l$ are both in $C^{1}([0, \infty) \times(0, \infty))$. Define the Banach functions spaces $\left(\mathcal{X},\|\cdot\|_{\mathcal{X}}\right)$ and $\left(\mathcal{Y},\|\cdot\|_{\mathcal{Y}}\right)$ by

$$
\begin{aligned}
& \mathcal{X}=\left\{W=W(u, r) \in C^{1}([0, \infty) \times(0, \infty)):\|W\|_{\mathcal{X}}<\infty\right\}, \\
& \mathcal{Y}=\left\{W=W(u, r) \in C^{1}([0, \infty) \times(0, \infty)):\|W\|_{\mathcal{Y}}<\infty\right\},
\end{aligned}
$$

where $\|W\|_{\mathcal{X}}:=\sup _{u \geq 0, r>0}\left\{r^{-2}\left[(1+r+u)^{4}|W(u, r)|+(1+r+u)^{5}\left|W^{\prime}(u, r)\right|\right]\right\}$, $\|W\|_{\mathcal{Y}}:=\sup _{u \geq 0, r>0}\left[r^{-2}(1+r+u)^{4}|W(u, r)|\right]$. It is now time to state and prove the main result of the work.

THEOREM 1. There exists a unique global classical solution $W \in C^{1}([0, \infty) \times(0, \infty))$ of $(31)$. In addition this solution fulfills the 
decay property

$$
|W(u, r)| \leq c(1+u+r)^{-2}, \quad\left|W^{\prime}(u, r)\right| \leq c(1+u+r)^{-3},
$$

where $c$ is a positive real constant. Moreover, the corresponding space-time is timelike and null geodesically complete toward the future.

Proof. Throughout the proof $\left(c_{i}\right)_{i=1,2, \ldots}$ denote positive real constants. Note that the functions spaces used here are fundamentally different from those of $[10,11,12,13]$. The cornerstone of the proof is the standard fixed point theorem. Define the mapping $\mathcal{K}: W \mapsto w=\mathcal{K}(W)$, where $w$ is the solution of the first order linear initial value problem

$$
D w=\frac{1}{2 r}(g-\widetilde{g})[w+(\sigma-I) \bar{W}], \quad w(0, r)=0 .
$$

$(i) \mathcal{K}$ is a mapping from a ball of $\mathcal{X}$ into itself. Let $x>0, W \in B_{x}$, where $B_{x}$ denotes the closed ball, in $\mathcal{X}$, of radius $x$ centered at 0 . From the definition of the differential operator $D$ in (31), the characteristic system of ODE associated to the initial value problem (34) is

$$
\frac{d r}{d u}=-\frac{1}{2} \widetilde{g}, \quad \frac{d w}{d u}=\frac{1}{2 r}(g-\widetilde{g})[w+(\sigma-I) \bar{W}],
$$

with initial data $r(0)=r_{0}, \quad w(0)=0$. Denote by $r(u)=\gamma\left(u, r_{0}\right)$ the solution of the Cauchy problem

$$
\frac{d r}{d u}=-\frac{1}{2} \widetilde{g}(u, r), \quad r(0)=r_{0}
$$

Then $r_{1}=r_{0}-\frac{1}{2} \int_{0}^{u_{1}} \widetilde{g}\left(u, \gamma\left(u, r_{0}\right)\right) d u$, where $r_{1}=\gamma\left(u_{1}, r_{0}\right)$. Integrating the second ODE of (35) along $\gamma$ we obtain

$$
w\left(u_{1}, r_{1}\right)=\int_{0}^{u_{1}}\left\{\exp \left(\int_{u}^{u_{1}}[N(u, r)]_{\gamma} d v\right)\right\}[f]_{\gamma} d u,
$$

where

$$
N(u, r)=\frac{1}{2 r}(g-\widetilde{g}), \quad f(u, r)=\frac{1}{2 r}(g-\widetilde{g})(\sigma-I) \bar{W} .
$$

We have to estimate $N$ and $f$. Since $W \in B_{x}$, the following estimates (39), (40) and (41) are straightforward from the definition of $\bar{W}$ in (26) and that of 
the Banach space $\left(\mathcal{X},\|\cdot\|_{\mathcal{X}}\right)$ in (32):

$$
\begin{gathered}
|W(u, r)| \leq \frac{x r^{2}}{(1+u+r)^{4}}, \\
|(\sigma-I) \bar{W}(u, r)| \leq|\bar{W}(u, r)| \leq \frac{x r^{2}}{3(1+u)(1+u+r)^{3}}, \\
|W(u, r)-\bar{W}(u, r)| \leq \frac{x r^{3}}{4(1+u)(1+u+r)^{4}} .
\end{gathered}
$$

(39) and (41) imply

$$
\left|\frac{1}{r}\left[(h-\bar{h})^{2}+\frac{1}{2} l^{2}\right]\right| \leq \frac{c_{1} x^{2} r^{3}}{(1+u)^{2}(1+u+r)^{6}} .
$$

This gives

$$
\int_{0}^{\infty}\left|\frac{1}{r}\left[(h-\bar{h})^{2}+\frac{1}{2} l^{2}\right]\right| d r \leq \frac{c_{2} x^{2}}{(1+u)^{4}}
$$

(42) yields

$$
\exp \left(-\int_{0}^{\infty}\left|\frac{1}{r}\left[(h-\bar{h})^{2}+\frac{1}{2} l^{2}\right]\right| d r\right) \geq \exp \left(-c_{2} x^{2}\right) .
$$

From (27), (28), the definition of $g$ and $\widetilde{g}$ in (31) and the estimate (41), we get

$$
|g(u, r)-\widetilde{g}(u, r)| \leq \frac{c_{3} x^{2} r^{4}}{(1+u)^{3}(1+u+r)^{5}} .
$$

In view of (43) and (44) we gain

$$
\widetilde{g}(u, r) \geq \exp \left(-c_{2} x^{2}\right)-c_{3} x^{2} .
$$

It is easy to see that the function $L=L(x)=\exp \left(-c_{2} x^{2}\right)-c_{3} x^{2}$ has a unique positive root $x_{0}$ and $L(x) \in(0,1]$ for all $x \in\left[0, x_{0}\right)$. We estimate the function $f$ defined in (38), by using (40) and (44), to get

$$
|f(u, r)| \leq \frac{17 x^{3} r^{2}}{480(1+u)^{4}(1+u+r)^{5}} .
$$


For $r(u)=\gamma\left(u, r_{0}\right)$ it holds, in view of (36) and (45), that

$$
r=r_{1}+\frac{1}{2} \int_{u}^{u_{1}} \widetilde{g}(s, r(s)) d s \geq r_{1}+\frac{L(x)}{2}\left(u_{1}-u\right) .
$$

Since $L(x) \in(0,1]$ for all $x \in\left[0, x_{0}\right),(47)$ implies

$$
1+u+r \geq 1+u+r_{1}+\frac{L(x)}{2}\left(u_{1}-u\right) \geq \frac{L(x)}{2}\left(1+u_{1}+r_{1}\right) .
$$

From (46) and (48) we gain the estimate

$$
\int_{0}^{u_{1}}\left|[f]_{\gamma}\right| d u \leq \frac{c_{4} x^{3} r_{1}^{2}}{\left(1+u_{1}+r_{1}\right)^{5} L^{5}(x)}
$$

In view of (44) we estimate the function $N$ defined in (38) to get

$$
|N(u, r)| \leq \frac{17 x^{2}}{160}
$$

From (50) we get

$$
\left|\int_{0}^{u_{1}}[N]_{\gamma} d v\right| \leq c_{2} x^{2}
$$

Considering (37), (49) and (51) we finally arrive at the following estimate for the solution $w=\mathcal{K}(W)$ of the initial value problem (34):

$$
\sup _{u \geq 0, r>0}\left[r^{-2}(1+u+r)^{4}|w(u, r)|\right] \leq \frac{c_{4} x^{3}}{L^{5}(x)} \exp \left(c_{2} x^{2}\right) .
$$

We now estimate $\sup _{u \geq 0, r>0}\left[r^{-2}(1+u+r)^{5}\left|w^{\prime}(u, r)\right|\right]$. Set $z(u, r)=w^{\prime}(u, r)$, with $z\left(0, r_{0}\right)=0$. Differentiating (34) w.r.t. $r$ yields

$$
D z=N_{1} z+B_{1}[w+(\sigma-I) \bar{W}]+N(\sigma-I) \bar{W}^{\prime},
$$

where $N_{1}=\frac{\widetilde{g}^{\prime}}{2}+\frac{g-\widetilde{g}}{2 r}, B_{1}=\frac{(g-\widetilde{g})^{\prime}}{2 r}-\frac{g-\widetilde{g}}{2 r^{2}}, N=\frac{g-\widetilde{g}}{2 r}$. By employing the same calculations and arguments as earlier we gain the following estimate for the solution $z$ of the initial value problem (53):

$$
\sup _{u \geq 0, r>0}\left[r^{-2}(1+r+u)^{5}|z(u, r)|\right] \leq \frac{\left(c_{5}+c_{6} x^{2}\right) x^{3}}{L^{10}(x)} \exp \left(c_{7} x^{2}\right) .
$$


It follows from (32), (52) and (54) that $\|w\|_{\mathcal{X}} \leq \frac{\left(c_{8}+c_{6} x^{2}\right) x^{3}}{L^{10}(x)} \exp \left(c_{7} x^{2}\right)$. To have $\|w\|_{\mathcal{X}} \leq x$, it suffices that $\Omega(x) \leq 1$, where $\Omega(x)=\frac{\left(c_{8}+c_{6} x^{2}\right) x^{2}}{L^{10}(x)} \exp \left(c_{7} x^{2}\right)$. One sees that $\lim _{x \rightarrow 0} \Omega(x)=0$. Hence there exists $x_{1} \in\left(0, x_{0}\right)$ such that, for $x \in\left(0, x_{1}\right], \Omega(x) \leq 1$. This shows that $\mathcal{K}$ is a mapping from $B_{x}$ into itself for $x \in\left(0, x_{1}\right]$.

(ii) The mapping $\mathcal{K}$ is a contraction. Let $w_{1}$ and $w_{2}$ be two solutions of (34) with $w_{j}(0, r)=0, w_{j}=\mathcal{K}\left(W_{j}\right), W_{j} \in \mathcal{X}, j=1,2$. We use the following shorthand notations $g_{j}=g\left(W_{j}\right), z_{j}=z\left(W_{j}\right)$, assume $\max \left\{\left\|W_{1}\right\|_{\mathcal{X}},\left\|W_{2}\right\|_{\mathcal{X}}\right\} \leq x \leq x_{1}$, and set for convenience $w_{1}-w_{2}=\vartheta$, $W_{1}-W_{2}=\boldsymbol{\Theta},\|\boldsymbol{\Theta}\|_{\mathcal{Y}}=y, D_{1}=\frac{\partial}{\partial u}-\frac{\widetilde{g_{1}}}{2} \frac{\partial}{\partial r}$. Then we have the following nonlinear initial value problem with unknown $\vartheta$

$$
D_{1} \vartheta=M[\vartheta+(\sigma-I) \bar{\Theta}]+B_{2}\left(w_{2}+(\sigma-I) \overline{W_{2}}\right)+B_{3} w_{2}^{\prime}, \quad \vartheta(0, r)=0,
$$

where $M=\frac{g_{1}-\widetilde{g_{1}}}{2 r}, B_{2}=\frac{\left(g_{1}-\widetilde{g_{1}}\right)-\left(g_{2}-\widetilde{g_{2}}\right)}{2 r}, B_{3}=\frac{\widetilde{g_{1}}-\widetilde{g_{2}}}{2}$. Using repeatedly the mean value theorem and similar tools as in the previous step we get $\|\mathcal{Q}\|_{\mathcal{Y}} \leq \Xi(x) y$, where $\Xi(x)=\frac{c_{9}\left(x^{2}+x^{4}\right)}{L^{4}(x)} \exp \left(c_{2} x^{2}\right)$. It is easy to see that the function $\Xi$ is strictly monotonically increasing on $\left(0, x_{1}\right]$ and $\lim _{x \rightarrow 0} \Xi(x)=0$. One deduces that there exists $x_{2} \in\left(0, x_{1}\right]$ such that $\Xi(x)<\frac{1}{2}$ for all $x \in\left(0, x_{2}\right]$. Thus the mapping $W \mapsto \mathcal{K}(W)$ contracts in $\mathcal{Y}$ for $\|W\|_{\mathcal{X}} \leq x_{2}$. This concludes the proof of the global existence and uniqueness of classical solution for (31). The decay property (33) of the solution is a direct consequence of the definition (32) of the Banach spaces $\left(\mathcal{X},\|\cdot\|_{\mathcal{X}}\right)$ and $\left(\mathcal{Y},\|\cdot\|_{\mathcal{Y}}\right)$.

In view of (27) and (42) one deduces easily from the definition of $g$ in (31) that, for each $r>0, g \longrightarrow 1$ if $u \longrightarrow \infty$. In view of (44), this implies that, for each $r>0, \widetilde{g} \longrightarrow 1$ if $u \longrightarrow \infty$. So, as $u \longrightarrow \infty$, the metric given in Bondi coordinates by (7) becomes the Minkowski metric. This completes the proof of Theorem 1.

REMARK 2. The present work can serve as a basis for future investigation on the issue of global existence of generalized solutions, with arbitrary initial data, for the considered electrovac EME model. 
Acknowledgments. This work was supported by the University of Pretoria and a Focus Area Grant from the National Research Foundation of South Africa. We express our appreciation to the anonymous referee whose valuable comments and suggestions have improved this article.

\section{REFERENCES}

[1] LichnerowicZ A. Relativistic Hydrodynamics and Magnetohydrodynamics, New York, Benjamin, 1967, 207 pp.

[2] Choquet-Bruhat Y. General Relativity and the Einstein Equations, New York, Oxford University Press, 2009, 812 pp.

[3] ANile A. M. Relativistic fluids and magneto-fluids: With applications in astrophysics and plasma physics, New York, Cambridge University Press, 1989, 348 pp.

[4] FonT J. A. Numerical Hydrodynamics and Magnetohydrodynamics in General Relativity, Living Rev. Relativity, 11, 2008, o 7, 131 pp.

[5] Pugliese D., J. A. Valiente Kroon. On the evolution equations for ideal magnetohydrodynamics in curved spacetime, arXiv:1112.1525, $25 \mathrm{pp}$.

[6] Agathonov A. A., Y. G. Ignatyev. Exact solution of the relativistic magnetohydrodynamic equations in the background of a plane gravitational wave with combined polarization, Gravit. Cosmol., 17, 2011, No 1, 71-75.

[7] Etienne Z. B. et al. Relativistic magnetohydrodynamics in dynamical spacetimes: Improved electromagnetic gauge condition for adaptative mesh refinement grids, Phys. Rev. D, 85, 2012, No 2, 024013, 10 pp.

[8] Noutchegueme N., C. NANGne. Global Existence of Solution to the Coupled Einstein-Maxwell System for a Perfect Charged Relativistic Fluid in a Robertson-Walker Space-Time, Adv. Studies Theor. Phys., 2, 2008, No 15, $725-740$.

[9] Christodoulou D. The formation of black holes in general relativity, Zürich, EMS Monographs in Mathematics, 2009, 600 pp.

[10] Christodoulou D. The problem of a self-gravitating scalar field, Comm. Math. Phys., 105, 1986, 337-361.

[11] CHAE D. Global existence of solutions to the coupled Einstein and Maxwell-Higgs system in the spherical symmetry, Ann. Henri Poincaré, 4, 2003, 35-62.

[12] TADMON C. Global solutions, and their decay properties, of the spherically symmetric $\mathfrak{S} \mathfrak{H}(2)-$ Einstein-Yang-Mills-Higgs equations, C. R. Acad. Sci. Paris Ser. I, 349, 2011, 1067-1072.

[13] Tadmon C., S. B. TchaPNDA. On the spherically symmetric Einstein-YangHiggs equations in Bondi coordinates, Proc. R. Soc. A, 468, 2012, 31913214.

[14] Dossa M., C. Tadmon. The Goursat problem for the Einstein-Yang-MillsHiggs system in weighted Sobolev spaces, C. R. Acad. Sci. Paris Ser. I, 348, $2010,35-39$. 
[15] Dossa M., C. TADmon. The characteristic initial value problem for the Einstein-Yang-Mills-Higgs system in weighted Sobolev spaces, Appl. Math. Res. Express. AMRX 2010, 2010, No 2, 154-231.

[16] Rendall A. D. Reduction of the characteristic initial value problem to the Cauchy problem and its applications to the Einstein equations, Proc. R. Soc. A, 427, 1990, 221-239.

[17] Tabensky R., A. H. Taub. Plane symmetric self-gravitating fluids with pressure equal to energy density, Comm. Math. Phys., 29, 1973, 61-77.

[18] Lefloch P. G., J. M . STEWART. The characteristic initial value problem for plane symmetric spacetimes with weak regularity, Classical Quantum Gravity, 28, 2011, No 14, 145019, $37 \mathrm{pp}$.

Manoscritto pervenuto in redazione il 20 Novembre 2012. 